\title{
Design of Dual- and Triple-Passband Filters Using Alternately Cascaded Multiband Resonators
}

\author{
Chi-Feng Chen, Ting-Yi Huang, and Ruey-Beei Wu, Senior Member, IEEE
}

\begin{abstract}
A novel method for designing multiband bandpass filters has been proposed in this paper. Coupling structures with both Chebyshev and quasi-elliptic frequency responses are presented to achieve dual- and triple-band characteristics without a significant increase in circuit size. The design concept is to add some extra coupled resonator sections in a single-circuit filter to increase the degrees of freedom in extracting coupling coefficients of a multiband filter and, therefore, the filter is capable of realizing the specifications of coupling coefficients at all passbands. To verify the presented concept, four experimental examples of filters with a dual-band Chebyshev, triple-band Chebyshev, dual-band quasi-elliptic, and triple-band quasi-elliptic response have been designed and fabricated with microstrip technology. The measured results are in good agreement with the full-wave simulation results.
\end{abstract}

Index Terms-Coupling coefficient, external quality factor, microstrip filter, stepped-impedance resonator, transmission zero.

\section{INTRODUCTION}

$\mathbf{I}$ N MODERN wireless and mobile communication systems, $\mathrm{RF} /$ microwave filters are always important and essential components. Planar filters are particularly popular structures because they can be fabricated using printed circuit technology and are suitable for commercial applications due to their compact size and low-cost integration [1]. Therefore, many applications to planar filters such as parallel- and cross-coupled bandpass filters have been extensively used in microwave communication systems due to their high-practicality, high-performance, and simple synthesis procedures [2]-[6].

Recently, in exploring advanced dual-band wireless systems, filters with dual-band operation for RF devices have become quite popular. Therefore, dual-band filters had been widely studied in several papers [7]-[17]. In [7], a dual-band filter was implemented by the combination of two individual filters with two specific single passbands. Extra impedance-matching networks must be used to design the input and output structure of the filter. In [8], three open stubs in parallel are introduced to create three transmission zeros for separating the two passbands. In [9], an optimization scheme based on hybrid-coded genetic-algorithm techniques is presented to design dual-band filters. However, physical meanings are less addressed in the design process. In [10], a dual-band bandpass filter is achieved by a cascade connection of a wideband bandpass filter and a bandstop filter, resulting in a large circuit size. Furthermore, dual-band resonators with open stubs in series and parallel [11],

Manuscript received April 12, 2006. This work was supported in part by the National Science Council under Grant NSC 93-2752-E-002-003-PAE.

The authors are with the Department of Electrical Engineering and Graduate Institute of Communication Engineering, National Taiwan University, Taipei, Taiwan 10617, R.O.C. (e-mail: d92942005@ntu.edu.tw).

Digital Object Identifier 10.1109/TMTT.2006.880653 dual-band stepped-impedance resonator filters [12]-[16], and a dual-band filter using coupled resonator pairs [17] are also presented to achieve dual-passband responses. With the rapid evolution of multiband and multiservice communication systems, filters with a multiband response will be largely required. Unfortunately, to our knowledge, triple-band filters have not been presented in past literature.

The aforementioned design techniques for dual-band filters based on a single filter circuit, however, are still challenging to the designer because it is difficult to exactly extract the desired coupling coefficients and external quality factors to simultaneously fit the specifications at both bands due to the limited degrees of freedom in the design parameters. Therefore, the concept of a dual-band filter based on a single circuit is quite difficult to extend to the design of triple-band filters. In order to conquer this problem without a significant increase in circuit size, this paper presents a new coupling structure by adding some extra coupled resonator sections to the single filter circuit. This novel structure can not only be used to design dual-band filters, but is also capable of designing triple-band filters. Both Chebyshev and quasi-elliptic response structures are proposed. Four experimental examples of filters with a dual-band Chebyshev, triple-band Chebyshev, dual-band quasi-elliptic, and triple-band quasi-elliptic response have been designed and implemented with microstrip technology to verify the proposed concept.

This paper is organized as follows. Section II describes the proposed design concept. Several new coupling structures for dual- and triple-band filters have been presented. Section III characterizes the theory of a stepped-impedance resonator. The design graphs for determining the structural parameters of a stepped-impedance resonator are provided. Sections IV-VII provide the design procedures for dual- and triple-band microstrip bandpass filters with Chebyshev and quasi-elliptic function responses. The experimental data are presented and compared with the simulated results. Finally, Section VIII draws some brief conclusions.

\section{Coupling Structures of DuAl- AND TRIPLE-PASSBAND FILTERS}

Fig. 1(a) shows the coupling structure of a dual-band parallelcoupled filter with an $N$ th-order response, where each node represents a resonator and the solid lines represent the direct coupling routes. The odd-number resonators are able to operate simultaneously at the center frequencies of the first and second passbands $\left(f_{1}\right.$ and $\left.f_{2}\right)$. The even-number resonators with superscripts I and II are able to operate only at $f_{1}$ and $f_{2}$, respectively. It should be noted that each pair of the even-number resonators operate at different frequencies, mutual coupling interference between them 


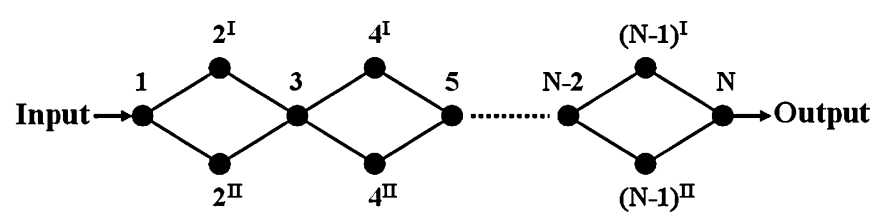

(a)

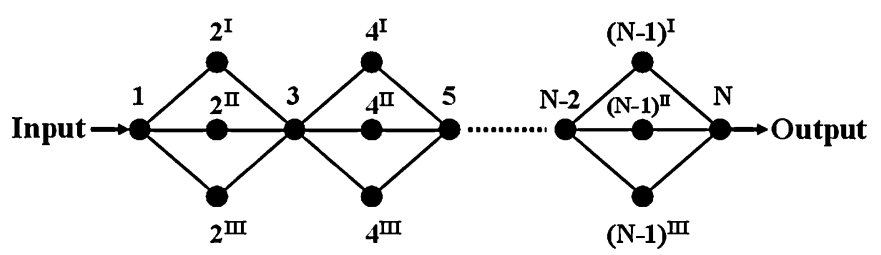

(b)

Fig. 1. Coupling structures of: (a) dual- and (b) triple-band parallel-coupled filters.

will not occur. This implies that each of the passbands can be implemented individually, i.e., each of the coupling coefficients between adjacent resonators can be extracted separately to meet the specifications. Degrees of freedom in extracting the coupling coefficients at the two specific passbands are then increased while compared to the conventional single-circuit dual-band filters. The design process is, therefore, more flexible and possible to be extended to achieve a multiband response.

Fig. 1(b) shows the extension of this concept, i.e., the coupling structure of a triple-band parallel-coupled filter with an $N$ th-order response. In a similar manner as Fig. 1(a), the oddnumber resonators are able to operate simultaneously at the center frequencies of the first, second, and third passbands $\left(f_{1}\right.$, $f_{2}$, and $f_{3}$ ), while the even-number resonators with superscripts I-III are designed to operate only at $f_{1}, f_{2}$, and $f_{3}$, respectively. As described above, each of the passbands in this structure can also be implemented individually. In other words, each of the coupling coefficients between adjacent resonators can be extracted separately at the three specific passbands.

In order to improve the selectivity of filters, the coupling structures of dual- and triple-band filters with a quasi-elliptic frequency response have also been proposed. Fig. 2(a) shows the coupling structure of a dual-band cross-coupled filter with a fourth-order response, where each node represents a resonator. The solid and dashed line represent the direct coupling and the cross-coupling routes, respectively. The multipath effect achieved by the combination of a direct coupling path and a cross-coupling path introduces a single pair of transmission zeros near the upper and lower sides of the passband edges at finite frequency, thus a much sharper filter skirt and higher selectivity can be achieved. In this structure, resonators 1 and 4 are able to operate simultaneously at $f_{1}$ and $f_{2}$, while the other resonators with superscripts I and II are designed to operate only at $f_{1}$ and $f_{2}$, respectively. As previously mentioned, each of the coupling coefficients between adjacent resonators can also be extracted separately at the two specific passbands. The same concept is also extended to the design of a triple-band quasi-elliptic filter, whose coupling structures are shown in Fig. 2(b).

It should be noted that the concept can be extended theoretically to design a filter with more passbands, however, problems caused by insufficient degrees of freedom of design parameters

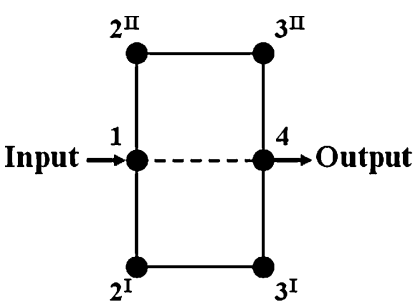

(a)

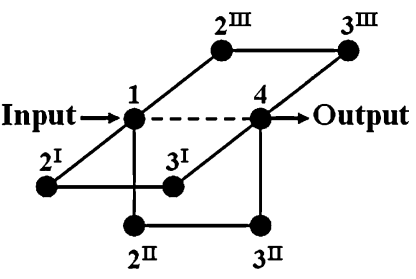

(b)
Fig. 2. Coupling structures of: (a) dual- and (b) triple-band cross-coupled filters with fourth-order response.

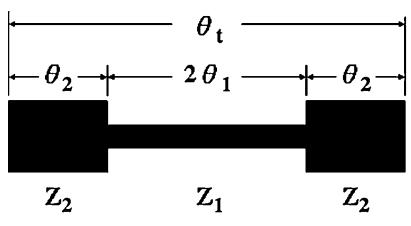

(a)

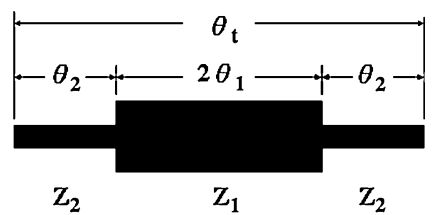

(b)
Fig. 3. Structure of the stepped-impedance resonator. (a) $K=Z_{2} / Z 1<1$. (b) $K=Z_{2} / Z_{1}>1$.

will arise in an actual design. For example, in the design of an input/output coupling structure, this will cause the restriction in designing the bandwidth of each passband. Moreover, in order to construct the odd-number resonators in Fig. 1 and resonators 1 and 4 in Fig. 2 for the simultaneous resonance at two or three specific frequencies, a simple kind of resonator that can control all the resonant frequencies, i.e., a stepped-impedance resonator, is used. This also limits the range of passband center frequency ratios if too many resonant frequencies are needed to be simultaneously controlled. Detailed descriptions for a steppedimpedance resonator will be presented in Section III.

\section{Characteristics OF STEPPED-IMPEDANCE Resonators}

The stepped-impedance resonator was originally presented not only to control spurious responses, but also to reduce resonator size [18], [19]. Fig. 3(a) and (b) shows the typical structures of the half-wavelength stepped-impedance resonator for the cases of $K<1$ and $K>1$, respectively, where $K$ is the impedance ratio defined as

$$
K=\frac{Z_{2}}{Z_{1}}
$$

If the length ratio $\alpha$ of the stepped-impedance resonator is defined as

$$
\alpha=\frac{\theta_{2}}{\theta_{1}+\theta_{2}}
$$

substituting (1) and (2) into the resonance conditions of the stepped-impedance resonator yields

and

$$
K \cdot \cot \left(\frac{1}{2} \alpha \cdot \theta_{t}\right)=\tan \left[\frac{1}{2}(1-\alpha) \cdot \theta_{t}\right]
$$

$$
K \cdot \cot \left(\frac{1}{2} \alpha \cdot \theta_{t}\right)=-\cot \left[\frac{1}{2}(1-\alpha) \cdot \theta_{t}\right]
$$




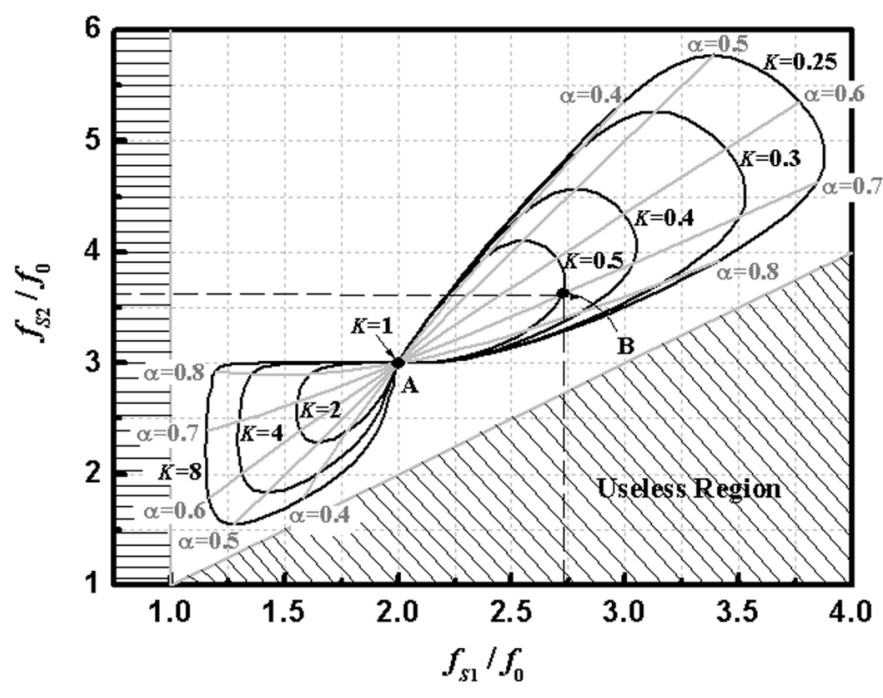

Fig. 4. Design graph for stepped-impedance resonators.

where (3) and (4) correspond to the odd- and even-mode resonances, respectively. It should be noted that the fundamental frequency and the other higher order mode frequencies can be determined by properly choosing a suitable combination of the impedance and the length ratios of the stepped-impedance resonator.

Fig. 4 shows the design graph for stepped-impedance resonators. Ratios of the first and second higher order resonant frequencies to the fundamental frequency $\left(f_{S 1} / f_{0}\right.$ and $\left.f_{S 2} / f_{0}\right)$ for half-wavelength stepped-impedance resonators are plotted with different values of $K$ and $\alpha$. It can be observed that, with typical values of $K$ and $\alpha$, realizable $f_{S 1} / f_{0}$ is between 1.2-3.8, and realizable $f_{S 2} / f_{0}$ is between 1.5-5.8. The case of $K=1$, i.e., point $\mathrm{A}$ in Fig. 4, denotes a traditional half-wavelength unit-impedance resonator whose spurious frequencies are the multiples of its fundamental frequency.

For example, if a resonator with $f_{S 1} / f_{0}=2.72$ and $f_{S 2} / f_{0}=3.62$ are required, one can locate point $\mathrm{B}$ with $K=0.5$ and $\alpha=0.7$ to satisfy the given specifications. It is also evident from Fig. 4 that $f_{S 1} / f_{0}>2$ and $f_{S 2} / f_{0}>3$ for the cases of $K<1$. The smaller the impedance ratio $K$ is, the larger $f_{S 1} / f_{0}$ and $f_{S 2} / f_{0}$ will be. On the contrary, if $f_{S 1} / f_{0}<2$ or $f_{S 2} / f_{0}<3$ is required, then the cases of $K>1$ must be chosen.

As described in Section II, resonators that are able to operate simultaneously at two or three specific frequencies are demanded in the design processes. Since the objective of our design is to look for this kind of resonators, Fig. 4 is quite useful for designing dual- or triple-mode resonators. As a result, when two or three resonant frequencies of a resonator are specified, a suitable combination of $K$ and $\alpha$ for the stepped-impedance resonator can be easily chosen from Fig. 4 and the dimensions of the resonator can, therefore, be determined.

\section{Design of a Dual-Passband Chebyshev Filter}

To demonstrate the usefulness of the proposed design concept for a multipassband filter, a dual-passband filter with a third-order Chebyshev frequency response and 0.1-dB ripple

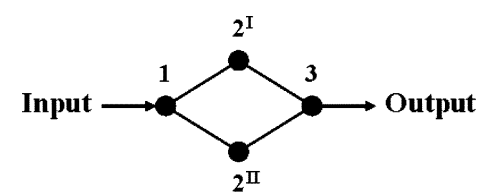

(a)

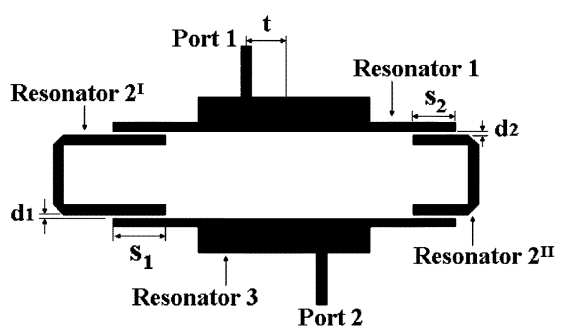

(b)

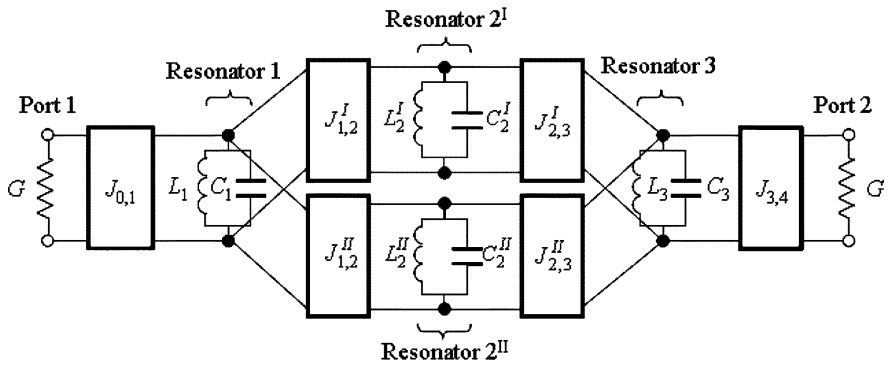

(c)

Fig. 5. (a) Coupling structure, (b) schematic layout, and (c) equivalent circuit of the dual-band Chebyshev filter.

level was designed and fabricated with the following specifications. The center frequencies of two bands are $f_{1}=2.8 \mathrm{GHz}$ and $f_{2}=4.2 \mathrm{GHz}$. The lower and higher fractional bandwidths are $\Delta_{1}=3.6 \%$ and $\Delta_{2}=4 \%$, respectively. The filter was designed to be fabricated using copper metallization on a Rogers RO4003 substrate with a relative dielectric constant of 3.38, a thickness of $0.508 \mathrm{~mm}$, and a loss tangent of 0.002 . Fig. 5(a) and (b) shows the coupling structure and circuit configuration of the filter. The equivalent-circuit model of the filter is shown in Fig. 5(c). The admittance inverter represents the coupling between resonators, where $J$ denotes the characteristic admittance. The first and last resonators (resonators 1 and 3 ) have to operate simultaneously at 2.8 and $4.2 \mathrm{GHz}$ and, hence, their physical parameters can then be easily determined from Fig. 4. One of the suitable solutions is to choose the combinations of $K=2.7$ and $\alpha=0.5$. Besides, resonators $2^{\mathrm{I}}$ and $2^{\mathrm{II}}$ have to operate at 2.8 and $4.2 \mathrm{GHz}$, respectively. A half-wavelength unit-impedance resonator is employed to realize these two resonators and each of them are folded for a compact design.

Since filter order and ripple level are specified and identical at the two passbands, the value of $\Delta_{2} / \Delta_{1}$ can be obtained by

$$
\frac{\Delta_{2}}{\Delta_{1}}=\frac{Q_{e 1}}{Q_{e 2}}
$$

where subscript 1 and 2 denotes the first and second passbands, respectively. The external quality factor can be characterized by

$$
Q_{e}=\frac{f_{0}}{\delta f_{3-\mathrm{dB}}}
$$




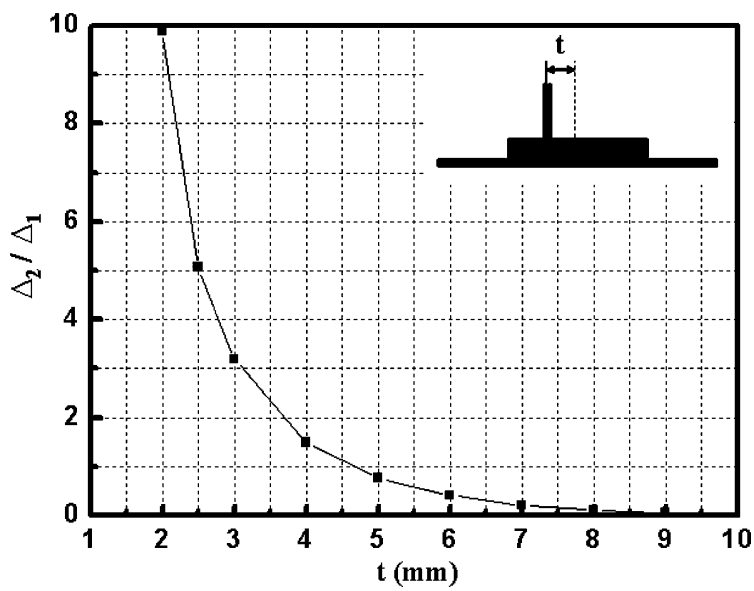

Fig. 6. Ratio $\Delta_{2} / \Delta_{1}$ versus the feeding point of input/output resonator.

where $f_{0}$ and $\delta f_{3-\mathrm{dB}}$ represent the resonant frequency and the $3-\mathrm{dB}$ bandwidth of the input or output resonator. The feeding points at the input and output resonators directly relate the filter bandwidth and external quality factor. Fig. 6 illustrates the simulated results of $\Delta_{2} / \Delta_{1}$ versus the feeding point of the input/ output resonator. As can be seen, once $\Delta_{2} / \Delta_{1}$ is specified, the feeding point can be easily determined. As indicated by this figure, when $t$ is adjusted from 2 to $9 \mathrm{~mm}$, a wide range of $\Delta_{2} / \Delta_{1}$, i.e., from 10 to 0.2 , can be obtained. It should be noted that, once this figure is reconstructed for specific substrates, the other procedures in this paper can be repeated directly.

As described in Section II, each of the passbands of the filter can be implemented individually. This is equivalent to the design of two single band filters independently, thus the traditional design procedure of a single band filter described in [5] can be followed directly. The design parameters of bandpass filter, i.e., the coupling coefficients and external quality factor, can be obtained from circuit elements of a low-pass prototype filter. The lumped circuit element values of the low-pass prototype filter are found to be $g_{0}=1, g_{1}=1.0315, g_{2}=1.1474$, $g_{3}=1.0315$, and $g_{4}=1$. In order to obtain the physical dimensions of the filter, the coupling coefficients and input/output external quality factors can then be found as $M_{12}^{\mathrm{I}}=M_{23}^{\mathrm{I}}=0.033$ and $Q_{e 1}=28.9$ for the first passband and $M_{12}^{\mathrm{II}}=M_{23}^{\mathrm{II}}=0.037$ and $Q_{e 2}=26$ for the second passband. A full-wave simulator has been used to extract the above parameters. When two synchronously tuned coupled resonators have a close proximity, the coupling coefficient can be evaluated from the two dominant resonant frequencies. If $f_{p 1}$ and $f_{p 2}$ are defined to be the lower and higher of the two resonant frequencies, respectively, the coupling coefficient can be obtained by

$$
M_{i j}=\frac{f_{p 2}^{2}-f_{p 1}^{2}}{f_{p 2}^{2}+f_{p 1}^{2}}
$$

where $M_{i j}$ represents the coupling coefficient between resonators $i$ and $j$.

The overall design procedure of this example can be summarized as follows. First, determine the dimensions of each resonator to meet the respective frequencies and then determine

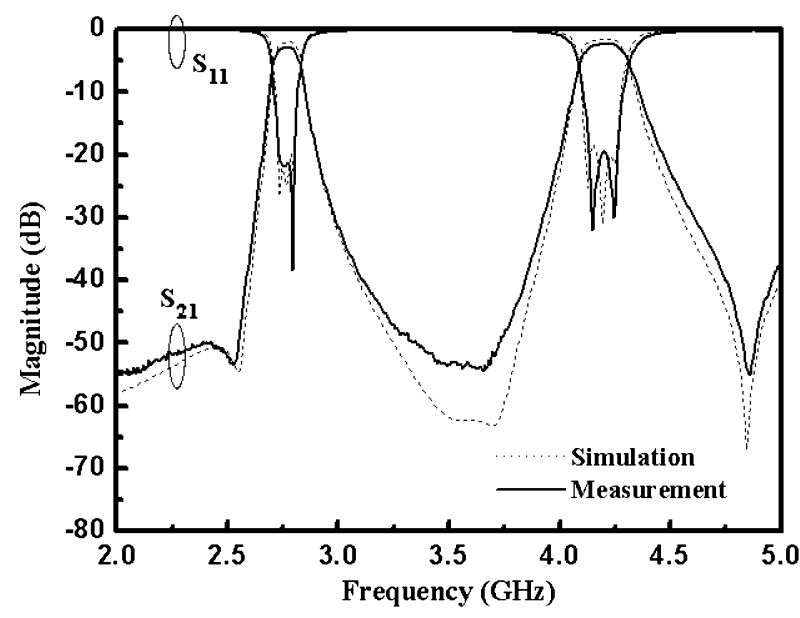

Fig. 7. Simulated and measured results of the dual-band Chebyshev filter.

the feeding position $t$ of the first/last resonator from Fig. 6 to meet the specific $\Delta_{2} / \Delta_{1}$ or $Q_{e}$. The final step is to adjust the coupling gaps $d_{1}$ and $d_{2}$, the overlapped lengths $s_{1}$ and $s_{2}$ to meet the specific coupling coefficients at the first and second passbands, respectively.

Realizable design geometric parameters have been obtained as $d_{1}=0.45 \mathrm{~mm}, d_{2}=0.45 \mathrm{~mm}, s_{1}=6.19 \mathrm{~mm}, s_{2}=$ $4.93 \mathrm{~mm}$, and $t=4.4 \mathrm{~mm}$. The prototype circuit size of the filter is approximately $49.6 \mathrm{~mm} \times 18.2 \mathrm{~mm}$, i.e., approximately $0.74 \lambda_{g}$ by $0.27 \lambda_{g}$, where $\lambda_{g}$ is the guided wavelength on the substrate at the center frequency of the first passband. Measurement was carried out using an Agilent E5071B network analyzer. The measured and simulated results of the filter are illustrated in Fig. 7. As can be seen, a transmission zero at the lower side of each passband edge is observed. Due to the cross coupling between nonadjacent resonators, the electrical behavior is similar to the traditional trisection filters, as described in [5], thus the selectivity on the lower side is better than that on the higher side at each passband. It is also obvious that two additional transmission zeros occur at approximately 3.5 and $4.8 \mathrm{GHz}$, which are the frequencies when the lengths between the tapped points and open-ends of the input/output resonators approximate a quarter guided wavelength. The measured lower and higher fractional bandwidths are $\Delta_{1}=3.6 \%$ and $\Delta_{2}=4 \%$, respectively. The measured passband return losses at both bands are below $-19 \mathrm{~dB}$, while the passband insertion losses are approximately 2.9 and $2.3 \mathrm{~dB}$ at the first and second passbands, respectively. The insertion losses would be attributed mainly to the conductor and dielectric losses. The measured results are in good agreement with the simulated predictions.

\section{Design of a Dual-Passband Quasi-Elliptic Filter}

In order to improve the selectivity of dual-band filter, a quasielliptic filter with a dual-passband response has been designed. The circuit configuration of the filter is shown in Fig. 8(a), and its coupling structure is shown in Fig. 2(a). Fig. 8(b) shows the equivalent circuit model of the filter. It is obvious from Fig. 8(a) that compact folded stepped-impedance resonators and U-shaped unit-impedance resonators are used as the building 


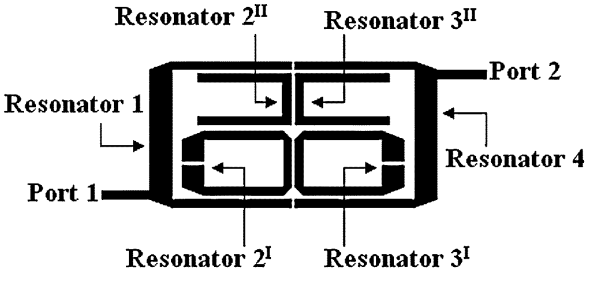

(a)

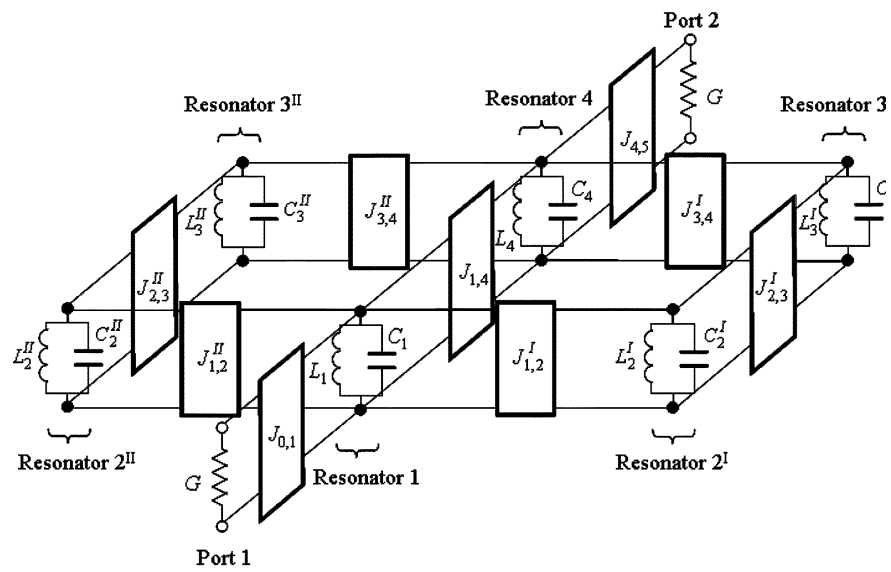

(b)

Fig. 8. (a) Schematic layout and (b) equivalent circuit of the dual-band quasielliptic filter.

block. This special arrangement of resonators helps not only to create the cross coupling between nonadjacent resonators, but also to reduce the circuit size. It can be anticipated that a single pair of transmission zeros near each passband at finite frequency will occur due to the multipath effect. In addition, the skew-symmetrical $\left(0^{\circ}\right)$ feeding structure [20] is introduced to realize the input and output ports of the filter. A filter with a skew-symmetrical feeding structure could create extra transmission zeros in the stopband. Thus, the selectivity and out-of-band rejection of the filter can be significantly improved.

The proposed filter is fabricated on the same Rogers RO4003 substrate as mentioned in the above section. The quasi-elliptic dual-band filter was designed with the following specifications. The center frequencies and fractional bandwidths are $f_{1}=2 \mathrm{GHz}, f_{2}=3.1 \mathrm{GHz}$, and $\Delta_{1}=\Delta_{2}=5.2 \%$, respectively. The lumped circuit element values of the low-pass prototype filter are found to be $g_{0}=1, g_{1}=0.9491$, $g_{2}=1.3508, J_{1}=-0.1173$, and $J_{2}=1.0119$. The required coupling coefficients and external quality factors are found to be $M_{12}=M_{34}=0.046, M_{23}=0.039, M_{14}=-0.006$, and $Q_{e}=18.3$ for the first and second passbands. As previously mentioned, both passbands can also be designed individually, therefore, a single band filter design can be applied based on the knowledge of the coupling coefficients of the three basic coupling structures, i.e., electric, magnetic, and mixed couplings, which has been well documented in [5].

In the same manner as Section IV, since the filter-order and lumped circuit element values of the low-pass prototype filter are specified and identical at the two passbands, $\Delta_{2} / \Delta_{1}$ can be obtained by (5). Fig. 9 illustrates $\Delta_{2} / \Delta_{1}$ versus the feeding

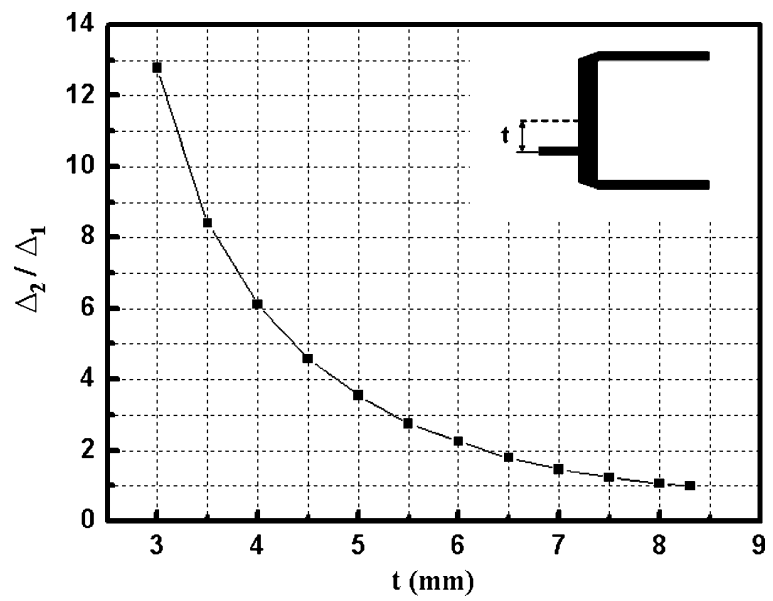

Fig. 9. Ratio $\Delta_{2} / \Delta_{1}$ versus the feeding point of input/output resonator.

point of the input/output resonator. Once $\Delta_{2} / \Delta_{1}$ is specified, the feeding point can be easily determined.

To implement this filter example, the first step is to determine the dimensions of each resonator to meet the respective frequencies. Resonators 1 and 4 based on a folded steppedimpedance resonator are designed to operate simultaneously at 2 and $3.1 \mathrm{GHz}$, resonators $2^{\mathrm{I}}$ and $3^{\mathrm{I}}$ based on a folded steppedimpedance resonator are design to operate at $2 \mathrm{GHz}$, and resonators $2^{\mathrm{II}}$ and $3^{\mathrm{II}}$ based on a U-shaped unit-impedance resonator are designed to operate at $3.1 \mathrm{GHz}$. The input and output feeding points $(t)$ are then selected for proper $\Delta_{2} / \Delta_{1}$ or $Q_{e}$. The last step is to individually determine the coupling gaps between resonators to match the specific coupling coefficients listed above.

Additionally, it is worth mentioning that when the gap between the nonadjacent resonators (resonators 1 and 4) is given, the corresponding coupling coefficients at two passbands are unlikely the same. Fortunately, the required coupling coefficients between nonadjacent resonators are very small, which are about one order less than the others between adjacent resonators. Thus, a slight error $(<20 \%)$ in this coupling coefficient hardly affects passband performance.

Fig. 10(a) presents a photograph of the filter. The size of the filter is $39.3 \mathrm{~mm} \times 20 \mathrm{~mm}$, i.e., only approximately $0.42 \lambda_{g}$ by $0.21 \lambda_{g}$, where $\lambda_{g}$ is the guided wavelength on the substrate at the center frequency of the first passband. The measured and simulated results of the filter are illustrated in Fig. 10(b). As expected, there is a single pair of transmission zeros near each passband due to cross-coupling effects, i.e., transmission zeros at approximately 1.9, 2.1, 2.9, and $3.2 \mathrm{GHz}$ can be observed. It can also be clearly observed that there are three extra transmission zeros at approximately 1.6, 2.45, and 3.6 GHz over the measured frequency range due to the skew-symmetrical feeding structure, resulting in a good stopband response. The measured lower and higher fractional bandwidths are $\Delta_{1}=5.3 \%$ and $\Delta_{2}=5.7 \%$, respectively. The measured passband return losses are both below $-15 \mathrm{~dB}$ at two passbands. The passband insertion losses are approximately 2.1 and $2.2 \mathrm{~dB}$ at the first and second passbands, respectively, which would be attributed 


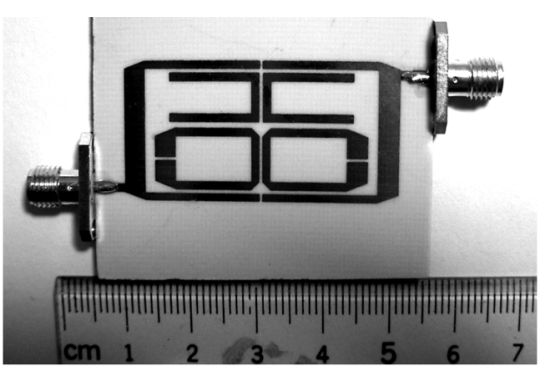

(a)

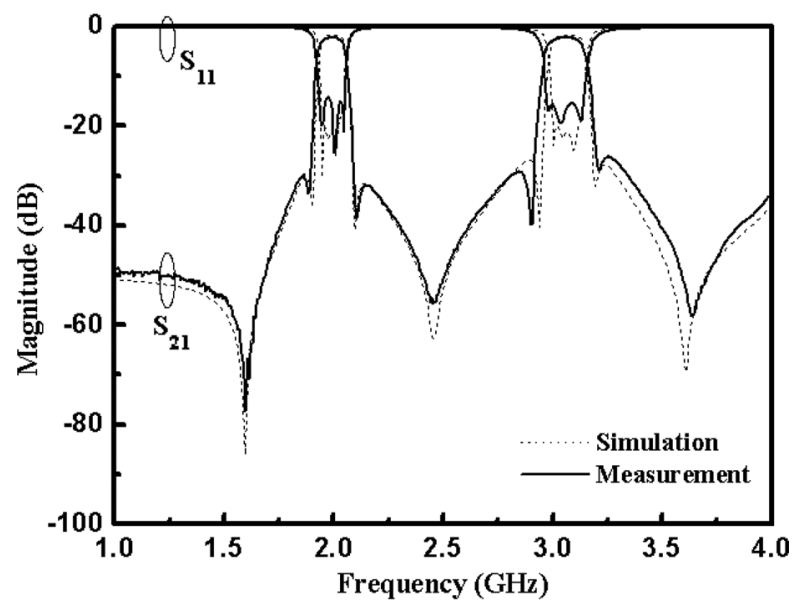

(b)

Fig. 10. (a) Fabricated filter. (b) Simulated and measured results of the dualband quasi-elliptic filter.

mainly to the conductor and dielectric losses. The measured results are in good agreement with the simulated predictions.

Compared with the dual-band quasi-elliptic filter in [15], there are three major advantages arising from the proposed configuration shown in Fig. 8. The first advantage is the flexible design process in our structure because it has increased the degrees of freedom in extracting the design parameters. The second one is the smaller circuit size in our structure due to the special arrangement of resonators. Moreover, extra impedance transformers at the input/output ports are required in [15], which are not needed in our design.

\section{Design of A TRIPle-PASSBAND QuASI-ElLIPTIC FILTER}

In this design example, a quasi-elliptic filter with a triple-passband response is realized with compact folded stepped-impedance resonators and U-shaped unit-impedance resonators as the building block. The circuit configuration of the filter is shown in Fig. 11(a), and its coupling structure is shown in Fig. 2(b). The equivalent-circuit model of the filter is shown in Fig. 11(b). Similarly, this special arrangement of resonators helps not only to create the cross-coupled effects, but also to reduce the circuit size. Also, it can be anticipated that a single pair of transmission zeros near each passband will occur due to the multipath effect and extra transmission zeros in the stopband will occur due to the skew-symmetrical feeding structure.

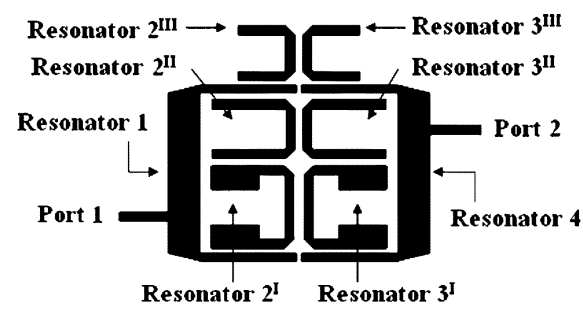

(a)

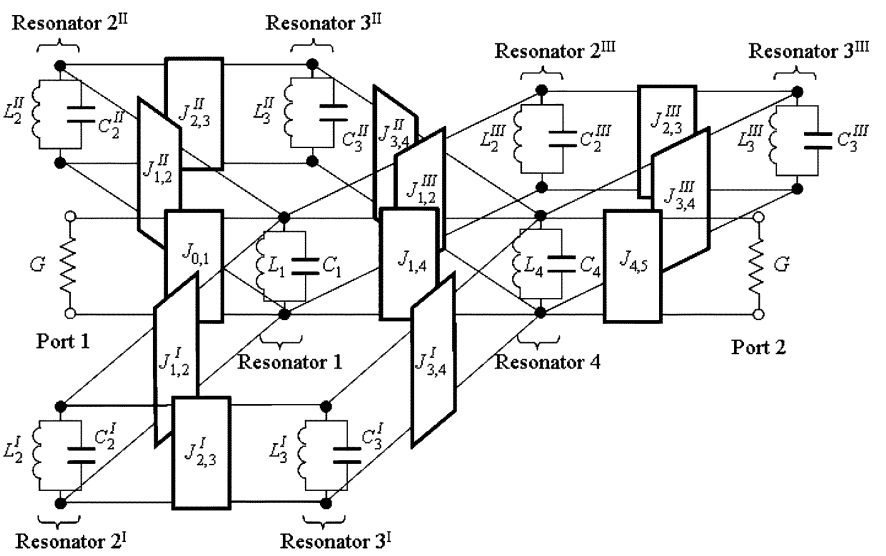

(b)

Fig. 11. (a) Schematic layout and (b) equivalent circuit of the triple-band quasielliptic filter.

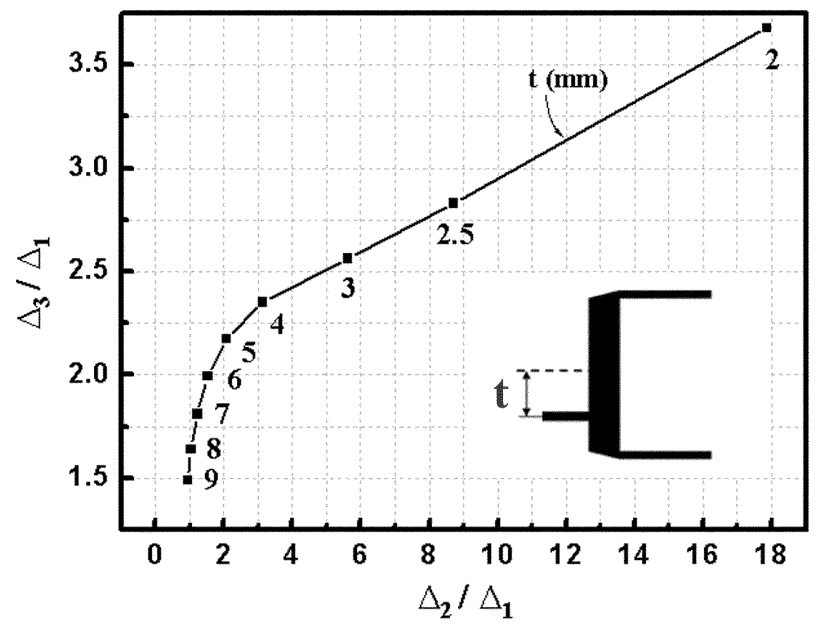

Fig. 12. Fractional bandwidth design graph for the filter.

Since filter order and lumped circuit element values of the low-pass prototype filter are specified and identical at the three passbands, the fractional bandwidth ratios can be written as

$$
\frac{\Delta_{2}}{\Delta_{1}}=\frac{Q_{e 1}}{Q_{e 2}} \text { and } \frac{\Delta_{3}}{\Delta_{1}}=\frac{Q_{e 1}}{Q_{e 3}}
$$

Fig. 12 illustrates the fractional bandwidth design graph for the filter. Obviously, once $\Delta_{2} / \Delta_{1}$ and $\Delta_{3} / \Delta_{1}$ are specified from the design curve, the feeding point can be easily determined. because there is only a single design parameter $t$ in designing the input/output tapped-line coupling structure, the range of realizable bandwidths is limited, as indicated. 
The proposed filter is fabricated on the same Rogers RO4003 substrate. The quasi-elliptic triple-band filter was designed with the following specifications. The center frequencies of three passbands are $f_{1}=2.5 \mathrm{GHz}, f_{2}=3.6 \mathrm{GHz}$, and $f_{3}=5.1 \mathrm{GHz}$, and the fractional bandwidths are $\Delta_{1}=4 \%, \Delta_{2}=3.8 \%$, and $\Delta_{3}=6 \%$. The same lumped circuit element values of the low-pass prototype filter, as mentioned in Section V, are used to design the filter. The coupling coefficients and the external quality factors are found to be $M_{12}^{\mathrm{I}}=M_{34}^{\mathrm{I}}=0.035, M_{23}^{\mathrm{I}}=$ $0.03, M_{14}^{\mathrm{I}}=-0.005$, and $Q_{e 1}=23.7$ for the first passband, $M_{12}^{\mathrm{II}}=M_{34}^{\mathrm{II}}=0.034, M_{23}^{\mathrm{II}}=0.028, M_{14}^{\mathrm{II}}=-0.005$, and $Q_{e 2}=25$ for the second passband, and $M_{12}^{\mathrm{III}}=M_{34}^{\mathrm{III}}=0.053$, $M_{23}^{\mathrm{III}}=0.045, M_{14}^{\mathrm{III}}=-0.007$, and $Q_{e 3}=15.8$ for the third passband.

The design procedures of this example are as follows. The first step is to determine the dimensions of each resonator to meet the respective frequencies. Resonators 1 and 4 based on a folded stepped-impedance resonator are designed to operate simultaneously at $2.5,3.6$, and $5.1 \mathrm{GHz}$, resonators $2^{\mathrm{I}}$ and $3^{\mathrm{I}}$ based on a folded stepped-impedance resonator are designed to operate at $2.5 \mathrm{GHz}$, resonators $2^{\mathrm{II}}$ and $3^{\mathrm{II}}$ based on a U-shaped unit-impedance resonator are designed to operate at $3.6 \mathrm{GHz}$, and resonators $2^{\mathrm{III}}$ and $3^{\mathrm{III}}$ based on a U-shaped unit-impedance resonator are designed to operate at $5.1 \mathrm{GHz}$. The second step is to determine the feeding position $t$ of the first/last resonator from Fig. 12 to meet the specific $\Delta_{2} / \Delta_{1}$ and $\Delta_{3} / \Delta_{1}$. The final step is to individually determine the coupling gaps between resonators to match the specific coupling coefficients listed above.

Fig. 13(a) presents a photograph of the filter. The size of the filter is $32.5 \mathrm{~mm} \times 29.1 \mathrm{~mm}$, i.e., only approximately $0.44 \lambda_{g}$ by $0.39 \lambda_{g}$, where $\lambda_{g}$ is the guided wavelength on the substrate at the center frequency of the first passband. The measured and simulated results of the filter are illustrated in Fig. 13(b). As expected, there are a single pair of transmission zeros near each passband due to cross-coupled effects, which can be observed at approximately $2.4,2.6,3.5,3.7,4.7$, and $5.5 \mathrm{GHz}$. It can also be clearly observed that there are three extra transmission zeros at approximately $2.1,2.9$, and $4.2 \mathrm{GHz}$ over the measured frequency range due to the skew-symmetrical feeding structure, resulting in a good stopband response. The measured fractional bandwidths are $\Delta_{1}=4 \%, \Delta_{2}=4 \%$, and $\Delta_{3}=6 \%$. The measured passband return losses are all below $-17 \mathrm{~dB}$, while the passband insertion losses are approximately $2.9,2.7$, and $2.3 \mathrm{~dB}$ at the first, second, and third passbands, respectively. Again, the insertion losses would be attributed mainly to the conductor and dielectric losses. The measured results are in good agreement with the simulated predictions. As a result, the triple-band quasi-elliptic filter with the advantages of compact size and high selectivity is quite useful for multiband and multiservice applications in future mobile communication systems.

\section{Design of A TriPle-PAssband Chebyshev Filter}

In order to overcome the problem of limited range of bandwidths in Section VI, the coupled-line coupling structure is employed to design the input/output coupling structure because it has more degrees of freedoms in the design process. Therefore, in the last design example, a triple-passband filter based

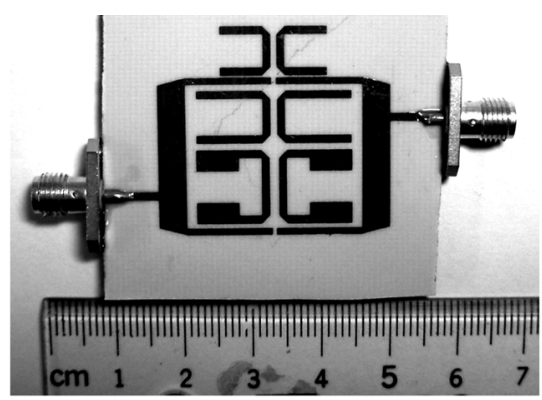

(a)

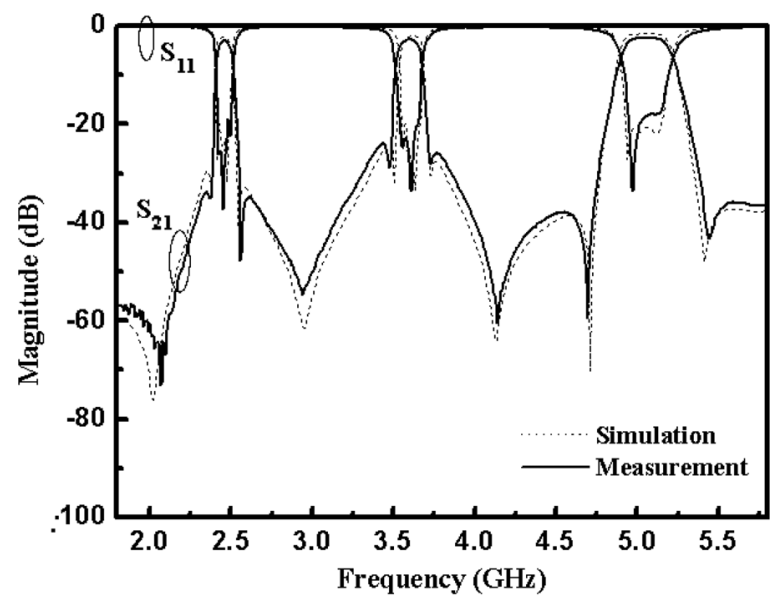

(b)

Fig. 13. (a) Fabricated filter. (b) Simulated and measured results of the tripleband quasi-elliptic filter.

on coupled-line coupling structure with third-order Chebyshev frequency response and a $0.1-\mathrm{dB}$ ripple level was designed and fabricated with the given specifications. The center frequencies of three bands are $f_{1}=2.3 \mathrm{GHz}, f_{2}=3.7 \mathrm{GHz}$, and $f_{3}=5.3 \mathrm{GHz}$. The fractional bandwidths are $\Delta_{1}=3.9 \%$, $\Delta_{2}=7 \%$, and $\Delta_{3}=5.2 \%$. Fig. 14(a) and (b) shows the coupling structure and circuit configuration of the filter. The equivalent-circuit model of the filter is shown in Fig. 14(c). As can be seen, the input and output are coupled through coupled lines to resonators 1 and 3 , and there are three design parameters $(w, L$, and $g$ ) in designing the coupled-line feeding structure. Thus, this solution is able to provide more choices in designing the bandwidths for three passbands.

The filter is designed to be fabricated on a Rogers RO4003 substrate with a thickness of $1.524 \mathrm{~mm}$. Fig. 15 illustrates the fractional bandwidth ratio design graph for the filter with $w=$ $5 \mathrm{~mm}$, which is the simulated results evaluated from (8). More design data can be obtained by choosing different combinations of $w, L$, and $g$. As a result, there are more choices in designing the bandwidths for three passbands when compared with that in Fig. 12. It should be noted that, when the substrate or filter structure is changed, the design graph should be restructured. However, once reconstructed, other procedures in this section can be followed directly.

The same lumped circuit element values of the low-pass prototype filter, as mentioned in Section IV, are used to design the filter. Thus, the coupling coefficients and single-loaded external quality factors of the triple-band filter can then be found to be 


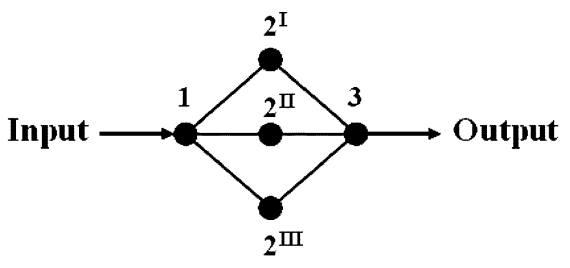

(a)

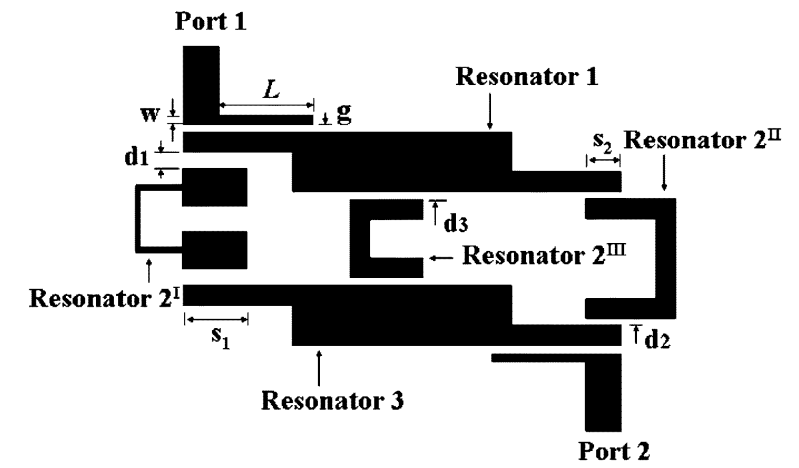

(b)

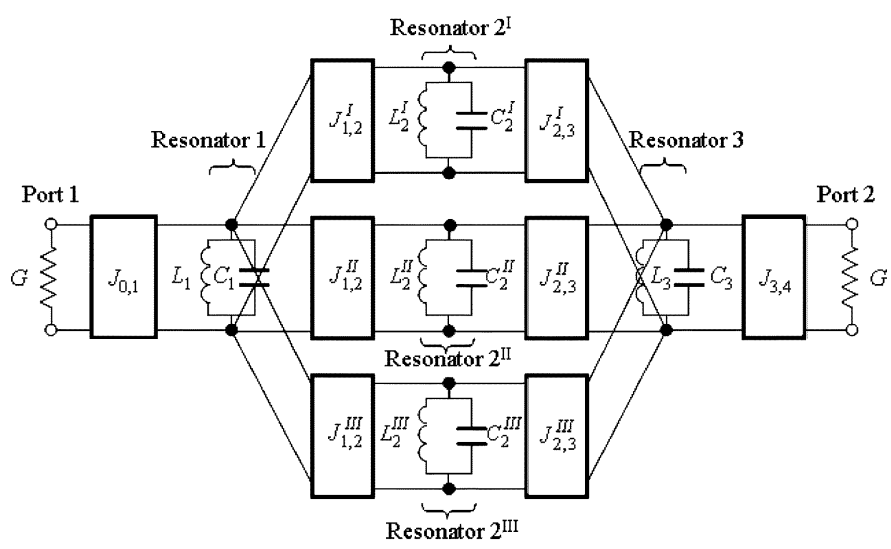

(c)

Fig. 14. (a) Coupling structure, (b) schematic layout, and (c) equivalent circuit of the triple-band Chebyshev filter.

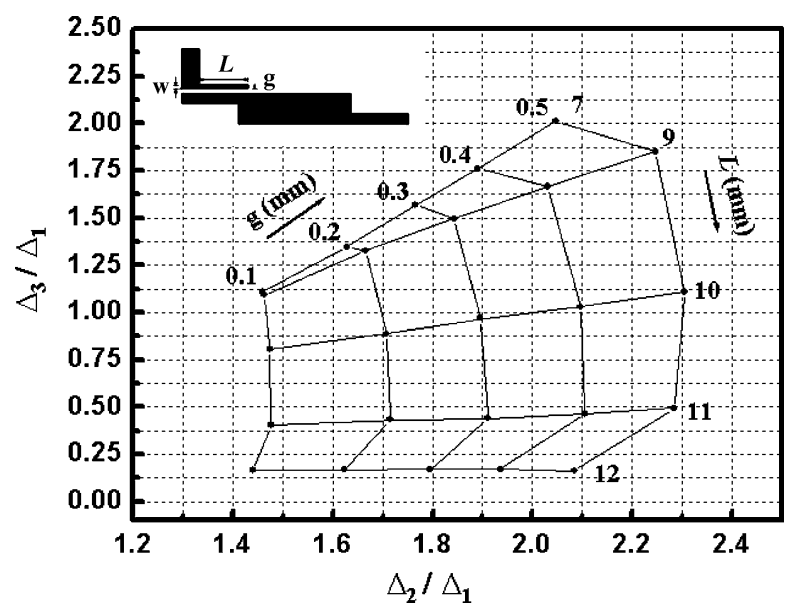

Fig. 15. Fractional bandwidth design graph for the filter.

$M_{12}^{\mathrm{I}}=M_{23}^{\mathrm{I}}=0.036$ and $Q_{e 1}=26.6$ for the first passband, $M_{12}^{\mathrm{II}}=M_{23}^{\mathrm{II}}=0.065$ and $Q_{e 2}=14.7$ for the second passband, and $M_{12}^{\mathrm{III}}=M_{23}^{\mathrm{III}}=0.048$ and $Q_{e 3}=19.9$ for the third passband.

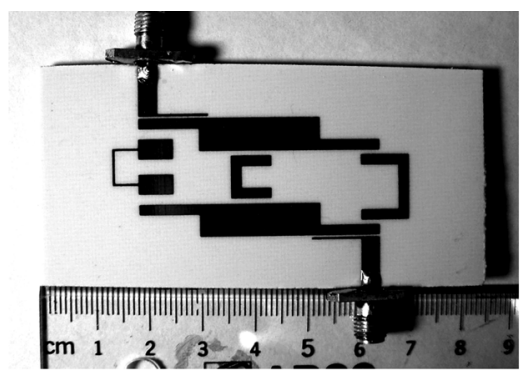

(a)

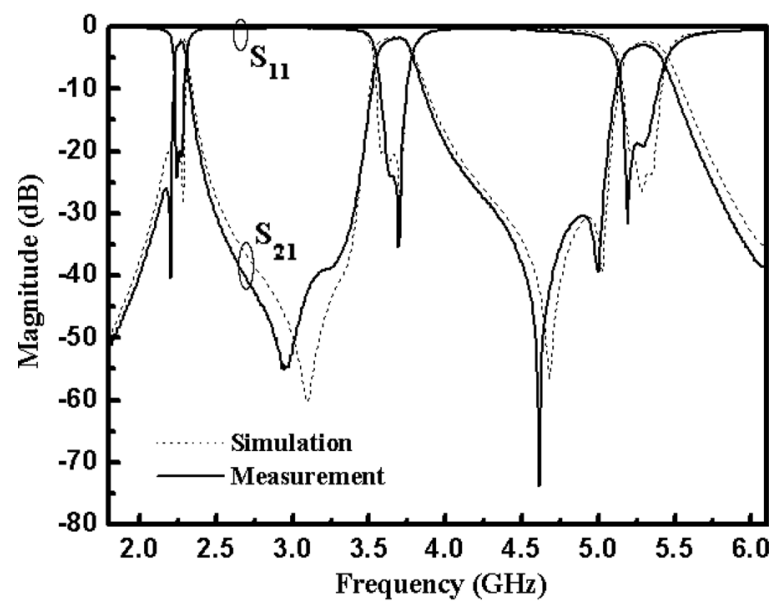

(b)

Fig. 16. (a) Fabricated filter. (b) Simulated and measured results of the tripleband Chebyshev filter.

The design procedure of this example is to at first determine the dimensions of each resonator to meet the respective frequencies. The first and last resonators (resonators 1 and 3) are designed to operate simultaneously at $2.3,3.7$, and $5.3 \mathrm{GHz}$. Their physical parameters can be determined by finding a combination of $K$ and $\alpha$ (in this example, $K=2$ and $\alpha=0.5$ ) from Fig. 4. Resonators $2^{\mathrm{II}}$ and $2^{\mathrm{III}}$ based on a folded unit-impedance resonator are designed to operate at 3.7 and $5.3 \mathrm{GHz}$, respectively. Resonator $2^{\mathrm{I}}$ is designed to operate at $2.3 \mathrm{GHz}$, which is based on a folded stepped-impedance resonator due to compactness consideration. The second step is to determine the coupled-line feeding structure at the input/output of the first/last resonator from Fig. 15 to satisfy the specific $\Delta_{2} / \Delta_{1}$ and $\Delta_{3} / \Delta_{1}$. Thirdly, determine the coupling gap $d_{3}$ to match the specific coupling coefficient of the third passband and, finally, adjust the parameters of $d_{1}$ and $s_{1}, d_{2}$ and $s_{2}$ to match the specific coupling coefficients of the first and second passbands, respectively.

Fig. 16(a) is the photograph of the filter. Geometric parameters for the filter are $d_{1}=1.72 \mathrm{~mm}, d_{2}=0.72 \mathrm{~mm}, d_{3}=$ $0.82 \mathrm{~mm}, s_{1}=6.38 \mathrm{~mm}, s_{2}=3.55 \mathrm{~mm}, L=9.22 \mathrm{~mm}$, $g=0.25 \mathrm{~mm}$, and $w=0.5 \mathrm{~mm}$. The resulting circuit size is approximately $54.1 \mathrm{~mm} \times 22.9 \mathrm{~mm}$, i.e., approximately $0.68 \lambda \mathrm{g}$ by $0.28 \lambda g$, where $\lambda g$ is the guided wavelength on the substrate at the center frequency of the first passband. The measured and simulated results of the filter are illustrated in Fig. 16(b). A transmission zero at the lower side of each passband is also observed, which is due the cross coupling between nonadjacent resonators. The measured fractional bandwidths are $\Delta_{1}=$ $3.8 \%, \Delta_{2}=6.8 \%$, and $\Delta_{3}=5 \%$. The measured passband return losses are at least below $-18 \mathrm{~dB}$, and the passband insertion 
losses are approximately $2.5,1.9$, and $2.9 \mathrm{~dB}$ at the first, second, and third passbands, respectively. The insertion losses would be attributed mainly to the conductor and dielectric losses. The measured results are again in good agreement with the simulated predictions.

\section{CONCLUSION}

In this paper, novel coupling structures have been presented to design dual- and triple-band microstrip bandpass filters without a significant increase in circuit size. Both theory and experiments have been provided. Several layouts of filters with dualband Chebyshev, dual-band quasi-elliptic, triple-band Chebyshev, and triple-band quasi-elliptic response have been designed and implemented with microstrip technology to demonstrate the proposed concepts. Different input/output coupling structures have been designed and compared in the two triple-band filter examples, showing that a filter with the coupled-line feeding structure is able to provide more choices in designing bandwidths. For the four examples, the measured results are all in good agreement with simulated predictions. No extra matching networks were needed for all four filters. As a result, these compact-size filter circuits are particularly suitable for multiband and multiservice applications in future mobile communication systems.

\section{REFERENCES}

[1] D. M. Pozar, Microwave Engineering, 2nd ed. New York: Wiley, 1998 , ch. 8.

[2] S. B. Cohn, "Parallel-coupled transmission-line-resonator filters," IEEE Trans. Microw. Theory Tech., vol. MTT-6, no. 4, pp. 223-231, Apr. 1958.

[3] E. G. Cristal and S. Frankel, "Hairpin-line and hybrid hairpin-line/halfwave parallel-coupled-line filters," IEEE Trans. Microw. Theory Tech., vol. MTT-20, no. 11, pp. 719-728, Nov. 1972.

[4] J. S. Hong and M. J. Lancaster, "Design of highly selective microstrip bandpass filters with a single pair of attenuation poles at finite frequencies," IEEE Trans. Microw. Theory Tech., vol. 48, no. 7, pp. 1098-1107, Jul. 2000.

[5] - Microstrip Filter for RF/Microwave Application. New York: Wiley, 2001.

[6] S. Y. Lee and C. M. Tsai, "New cross-coupled filter design using improved hairpin resonators," IEEE Trans. Microw. Theory Tech., vol. 48, no. 12 , pp. 2482-2490, Dec. 2000.

[7] H. Miyake, S. Kitazawa, T. Ishizaki, T. Yamada, and Y. Nagatom, "A miniaturized monolithic dual band filter using ceramic lamination technique for dual mode portable telephones," in IEEE MTT-S Int. Microw. Symp. Dig., 1997, vol. 2, pp. 789-792.

[8] C. Quendo, E. Rius, and C. Person, "An original topology of dual-band filter with transmission zeros," in IEEE MTT-S Int. Microw. Symp. Dig., 2003, vol. 2, pp. 1093-1096.

[9] M. I. Lai and S. K. Jeng, "Compact microstrip dual-band bandpass filters design using genetic-algorithm technique," IEEE Trans. Microw. Theory Tech., vol. 54, no. 1, pp. 160-168, Jan. 2006.

[10] L. C. Tsai and C. W. Hsue, "Dual-band bandpass filters using equallength coupled-serial-shunted lines and $Z$-transform technique," IEEE Trans. Microw. Theory Tech., vol. 52, no. 4, pp. 1111-1117, Apr. 2004.

[11] C. M. Tasi, H. M. Lee, and C. C. Tsai, "Planar filter design with fully controllable second passband," IEEE Trans. Microw. Theory Tech., vol. 53, no. 11, pp. 3429-3439, Nov. 2005.

[12] H. M. Lee, C. R. Chen, C. C. Tsai, and C. M. Tsai, "Dual-band coupling and feed structure for microstrip filter design," in IEEE MTT-S Int. Microw. Symp. Dig., 2004, vol. 3, pp. 1971-1974.

[13] S. Sun and L. Zhu, "Compact dual-band microstrip bandpass filter without external feed," IEEE Microw. Wireless Compon. Lett., vol. 15, pp. 644-646, Oct. 2005.
[14] M. L. Chuang, "Concurrent dual band filter using single set of microstrip open-loop resonators," Electron. Lett., vol. 41, pp. 1013-1014, Sep. 2005.

[15] J. T. Kuo and H. S. Cheng, "Design of quasi-elliptic function filters with a dual-passband response," IEEE Microw. Wireless Compon. Lett., vol. 14, pp. 472-474, Oct. 2004.

[16] J. T. Kuo, T. H. Yeh, and C. C. Yah, "Design of microstrip bandpass filters with a dual-passband response," IEEE Trans. Microw. Theory Tech., vol. 53, no. 4, pp. 1331-1337, Apr. 2005.

[17] C. C. Chen, "Dual-band bandpass filter using coupled resonator pairs," IEEE Microw. Wireless Compon. Lett., vol. 15, no. 4, pp. 259-261, Apr. 2005.

[18] M. Makimoto and S. Yamashita, "Bandpass filters using parallel coupled stripline stepped impedance resonators," IEEE Trans. Microw. Theory Tech., vol. 28, no. 12, pp. 1413-1417, Dec. 1980.

[19] M. Sagawa, M. Makimoto, and S. Yamashita, "Geometrical structures and fundamental characteristics of microwave stepped-impedance resonators," IEEE Trans. Microw. Theory Tech., vol. 45, no. 7, pp. 1078-1085, Jul. 1997.

[20] C. M. Tsai, S. Y. Lee, and C. C. Tsai, "Performance of a planar filter using a $0^{\circ}$ feed structure," IEEE Trans. Microw. Theory Tech., vol. 50, no. 10 , pp. 2362-2367, Oct. 2002.

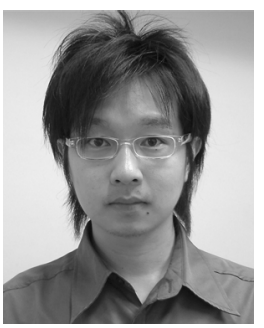

Chi-Feng Chen was born in PingTung, Taiwan, R.O.C., on September 3, 1979. He received the B.S. degree in physics from Chung Yuan Christian University, Taoyuan, Taiwan, R.O.C., in 2001, the M.S. degree in electrophysics from the National Chiao Tung University, Hsinchu, Taiwan, R.O.C., in 2003, and is currently working toward the Ph.D. degree in communication engineering at National Taiwan University, Taipei, Taiwan, R.O.C.

His research interests include the design of microwave filters and associated RF modules for microwave and millimeter-wave applications.

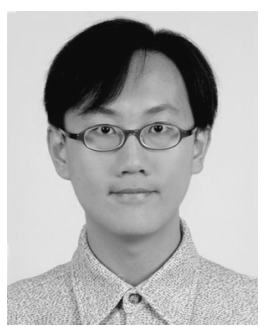

Ting-Yi Huang was born in Hualien, Taiwan, R.O.C., on November 12, 1977. He received the B.S. degree in electrical engineering and M.S. degree in communication engineering from National Taiwan University, Taipei, Taiwan, R.O.C., in 2000 and 2002 , respectively, and is currently working toward the $\mathrm{Ph} . \mathrm{D}$. degree in communication engineering at National Taiwan University.

His research interests include computational electromagnetics, the design of microwave filters, transitions, and associated RF modules for microwave and millimeter-wave applications.

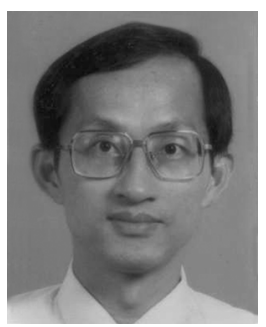

Ruey-Beei Wu (M'91-SM'97) received the B.S.E.E. and Ph.D. degrees from National Taiwan University, Taipei, Taiwan, R.O.C., in 1979 and 1985, respectively.

In 1982, he joined the faculty of the Department of Electrical Engineering, National Taiwan University, where he is currently a Professor. He is also with the Graduate Institute of Communications Engineering, which was established in 1997. From March 1986 to February 1987, he was a Visiting Scholar with IBM, East Fishkill, NY. From August 1994 to July 1995, he was with the Electrical Engineering Department, University of California at Los Angeles (UCLA). From 1998 to 2000, he was also appointed Director of the National Center for High-Performance Computing, and has served as Director of Planning and Evaluation Division since November 2002, both under the National Science Council. His areas of interest include computational electromagnetics, transmission line and waveguide discontinuities, microwave and millimeter-wave planar circuits, and interconnection modeling for computer packaging. 